\title{
Estudio sobre el cumplimiento de la normativa del ICAA para la calificación de las películas presentadas en el periodo 2005-2007
}

Study on Regulatory Compliance of the ICAA for Qualifying by Ages of the Films Presented in the Period 2005-2007

Juan José Muñoz García. CES Villanueva (UCM) y Universidad CEU San Pablo

Recibido: 9-X-2008 - Aceptado: 2-II-2009

Resumen:

El cine ejerce un papel indudable en la configuración de la sociedad. El espectador, por tanto, puede exigir que se protejan una serie de valores, sobre todo si son fundamentales para la formación de la infancia y la juventud. Para lograrlo es necesario que se respeten los códigos deontológicos vigentes. El objetivo principal de la presente investigación es evaluar la aplicación de los criterios de calificación por edades de las películas presentadas en el ICAA durante los años 2005-2007. Las conclusiones del estudio analizan los casos de incumplimiento y proponen una serie de medidas deontológicas para paliar esta situación.

Palabras clave:

Películas, ICAA, deontología, infancia, juventud, calificación por edades, comportamientos sociales, violencia, temática conflictiva, sexo.

Abstract:

The movies carry an obvious role in shaping society. The viewer can demand the protection of a range of values, especially if they are essential for the formation of children and youth. To achieve this it is necessary that the existing codes of ethics are respected. The main objective of this research is to evaluate the application of the criteria for qualifying by ages of the films presented at the ICAA for the years 2005-2007. The finding of the study analyzes the cases of default and proposes a series of ethical actions to alleviate this situation.

Key Words:

Movies, ICAA, Deontology, Infancy, Youth, Qualifying (rating) by Ages, Social Behavior, Violence, Thematic Conflictive, Sex. 


\section{Introducción}

El cine, junto con los demás medios audiovisuales, ejerce un papel indudable en la configuración de la sociedad. Se podría decir que miles de personas han alimentado su concepción del hombre y del mundo con los contenidos ofrecidos por los medios audiovisuales. Los deseos, proyectos y sueños de muchos individuos están poblados de imágenes publicitarias, televisivas y fílmicas. Mucha gente besa como se besa en el cine, o hace negocios como en el cine, o incluso mata como en el cine. No requiere mucho esfuerzo advertir que la sociedad de la comunicación nos ha ensanchado la realidad o, al menos, nuestra imagen de lo real, y ha ampliado nuestra experiencia virtual de la vida.

\section{La influencia social del cine}

La gran pantalla es una ventana mágica capaz de transportarnos a nuevos mundos, a otras épocas, países y personajes. Desde pequeños vivimos inmersos en esa máquina de los sueños. Gracias al séptimo arte reparamos en los múltiples detalles que forman la trama de nuestra existencia; por todo esto se puede considerar el cine como el principal educador de nuestros días (Marías, 1992: 212).

Ese potencial educador de la imagen juega un papel fundamental en la formación de la individualidad de los más jóvenes, de las ideas que obtienen acerca de las demás personas, y del lugar que pueden ocupar en el mundo. El cine y la televisión se transforman en agentes de socialización primaria, complementando o sustituyendo a otras instituciones (escuela, familia, Iglesia, etc.) (Arza, 2008: 9-11). Al mismo tiempo, y en comparación con los métodos educativos tradicionales -gracias a su capacidad para recrear de manera verosímil mundos reales o virtuales-, la pantalla consigue que los espectadores más vulnerables -niños y adolescentes- acaben concediendo una credibilidad tal a los contenidos audiovisuales que, en algunos casos, pueden ocasionar en estos chavales una total confusión y desconcierto con respecto a los más básicos criterios cognoscitivos y morales (Pérez, 2003: 28).

Los estudios realizados desde los años setenta hasta nuestros días confirman esta influencia social del cine y la televisión. Y aunque es imposible establecer una relación causa-efecto en este asunto, parece claro que los medios de comunicación no corrompen al hombre pero sí lo transforman. El cine nos suministra una determinada interpretación del mundo y recursos para justificar o legitimar creencias, actos e ideas (Pérez, 2003: 25-26). Tal influencia viene facilitada por el hecho de que nuestra personalidad se constituye de modo narrativo, pues desde tiempos inmemoriales el ser humano ha contado historias, relatos y mitos. Con ellos pretendía llegar a conocerse mejor y transmitir sus creencias, valores, miedos y proyectos. El séptimo arte, 
continuando esta antigua tradición, multiplicó de un modo insospechado la contribución de las narraciones al proceso de formación de la persona.

La función socializadora del cine y la televisión universaliza y multiplica los contenidos narrativos y dramáticos, dando lugar a un imaginario colectivo similar sobre asuntos que no conocemos de primera mano. Pero la industria audiovisual no sólo refleja lo que percibe en la realidad social, sino que también es capaz "de crear modelos de conducta, patrones culturales, que acaban siendo asumidos en algún grado por esa misma sociedad.” (Pardo, 2001: 130). En el uso de este poder radica la grave responsabilidad del profesional de lo audiovisual. Una responsabilidad superior a la de cualquier otro artista o intelectual.

Por todo ello, ya desde los inicios de la industria del cine, algunos directores y productores alertaron a la opinión pública sobre la grandeza y el riesgo de ese poder. "Es un pensamiento juicioso -afirma Cecil B. De Mille en su Autobiografía - creer que las decisiones que tomamos en los despachos de Hollywood pueden llegar a afectar a las vidas de seres humanos en todo el mundo". Y un productor creativo como David Puttnam (Carros de fuego, Un tipo genial (Local Hero), Los gritos del silencio o La Misión) nos recuerda que, "buenas o malas, las películas tienen un poder enorme: dan vueltas en el cerebro y se aprovechan de la oscuridad de la sala para formar o confirmar actitudes sociales" (Pardo, 2001: 135).

El espectador, por tanto, puede exigir que se protejan una serie de valores, sobre todo si son fundamentales para la formación de la infancia y la juventud. Ante esta responsabilidad claman muchos profesionales del mundo audiovisual, "que se sienten, a veces, inseguros sobre las consecuencias de su propio trabajo" (Pérez, 2003: 7) ${ }^{1}$.

Pero la responsabilidad pública de la comunicación exige que se respeten los códigos deontológicos vigentes, que son fruto de acuerdos en el ámbito de lo audiovisual. Asimismo, sería deseable que la autorregulación no busque únicamente el beneficio de quienes la promueven (cadenas de TV, productoras, distribuidoras, etc.), sino también el de los espectadores, que deben estar en su punto de mira a la hora de impulsar un cine de calidad (Muñoz, 2005: 35).

1 La cita continúa: "Se preguntan sobre si la creciente y obligada búsqueda de audiencia masiva no irá demasiado lejos: la espectacularización de la violencia, la potenciación de contenidos de transgresión, o la falta de respeto por la vida privada. Se preguntan, con intensidad más o menos silenciada, si el marco industrial y comercial en que desarrollan su tarea no acaba, a la larga, por menoscabar, en bastantes ocasiones, sus obligaciones éticas con respecto a la misión de servicio público y al desarrollo de la educación". Sobre este particular, véase también (Tur, 2006: 1-2, 42-43). 


\subsection{Los criterios del ICAA}

La normativa expuesta en el Ministerio de Cultura, a través del ICAA (Instituto de la Cinematografía y las Artes Audiovisuales), establece que toda película, en cualquier soporte, antes de ser comercializada debe presentarse en el ICAA o en la Comunidad Autónoma competente para su calificación por grupos de edad del público al que van destinadas las películas. La Comisión de Calificación de Películas Cinematográficas es el órgano asesor encargado del visionado de las mismas.

Las películas pueden ser calificadas según los siguientes grupos de edad:

- $\quad$ Especialmente recomendada para la infancia

- $\quad$ Para todos los públicos (T)

- $\quad$ No recomendada para menores de siete años (NR7)

- $\quad$ No recomendada para menores de trece años (NR13)

- $\quad$ No recomendada para menores de dieciocho años (NR18)

- $\quad$ Película X prohibido el acceso a menores de dieciocho años

Dicha calificación debe figurar visible en las taquillas de los cines o en las carátulas de los vídeos u otros soportes. Por otra parte, la calificación otorgada a una película tiene carácter orientativo, excepto las calificadas como películas X, que se exhibirán únicamente en salas especiales denominadas “Salas X”, y en caso de comercialización no cinematográfica deben presentarse al público en lugares especiales no accesibles a menores.

Para establecer con claridad esta valoración por edades de las películas que serán exhibidas en las salas de cine, se han establecido una serie de orientaciones deontológicas. Se trata de unos criterios otorgados por el citado Instituto de las Cinematografía y las Artes Audiovisuales (ICAA) que permiten clasificar las películas cinematográficas, en especial a las calificadas como no recomendadas para menores de trece años. Tales criterios también han sido asumidos en el reciente Código de autorregulación sobre contenidos televisivos e infancia ${ }^{2}$.

2 Dichos criterios pueden encontrarse en: www.tvinfancia.es. Debido a la limitación de espacio no podemos incluir aquí este texto del ICAA, pero tal vez sea conveniente su lectura, aunque no necesaria, para una mejor comprensión de nuestro estudio. 


\subsection{Beneficios de esta normativa: protección de la infancia y la adolescencia}

Esta normativa supone un acuerdo de autorregulación para el cine en España y una propuesta responsable por parte de la industria cinematográfica, que debe responder a la insatisfacción de muchos sectores sociales por la baja calidad de los contenidos de algunas películas estrenadas en nuestras salas.

Tales criterios suponen un compromiso voluntariamente adquirido ante la sociedad por parte del ICAA para demostrar a los espectadores que saben en qué consiste un cine de calidad y cuáles son los límites razonables que deben establecerse. Y que, por tanto, no era necesario imponer a los profesionales del séptimo arte controles externos a ellos mismos.

Un compromiso de "especial sensibilidad y cuidado" para con los menores, pues el gran principio que informa estas normas es establecer unos criterios mínimos para proteger a los niños y a los adolescentes a la hora de ir al cine.

La normativa del ICAA, aunque es orientativa, debe colaborar con los principales agentes educativos, de modo que haga más eficaz la labor de protección a la infancia y a la juventud. Para ello se requiere un comportamiento activo por parte de quienes tienen encomendada, de forma indirecta, la educación de niños y jóvenes, como es el caso de los medios audiovisuales.

Por todo esto, sería deseable que una parte especial de la producción cinematográfica no sólo estuviera libre de contenidos perjudiciales sino que tuviera un marcado carácter formativo, de modo que los padres pudieran contar con el cine como un agente de socialización fiable.

\section{Objetivo del informe: recuento y descripción de incumplimientos de la normativa del ICAA del año} 2005 al año 2007

A pesar de lo descrito en el apartado anterior, hay que lamentar que los convenios y acuerdos de autorregulación no siempre se cumplen como cabría esperar; una situación que afecta a la calidad de las cintas exhibidas en nuestras salas, y posteriormente editadas en DVD, emitidas por televisión o proyectadas en las aulas de colegios e institutos de educación secundaria.

Este es el contexto que ha suscitado el objetivo principal de la presente investigación: describir los incumplimientos o la deficiente aplicación de los criterios de la calificación por edades de las películas presentadas en el ICAA en España durante los años 2005, 2006 y 2007. 


\subsection{Metodología}

La metodología de trabajo empleada ha consistido en estudiar la base de datos del Instituto de la Cinematografía y las Artes Audiovisuales (http:/ /www.mcu.es/bbddpeliculas/buscarPeliculas.do) en la que se refleja las calificación de las películas registradas cada año y contrastar esa calificación con las normas dictadas por el propio ICAA (respecto a comportamientos sociales, violencia, temática conflictiva y sexo). Siempre que ha sido posible, también se ha establecido una comparación con la calificación otorgada por organismos similares de otros países y reflejada en la web www.imdb.com.

Se ha optado por realizar un análisis del contenido de los filmes que resultara más apropiado para un estudio de carácter ético-deontológico, por lo que nos hemos centrado principalmente en los temas de fondo de los filmes y en las conductas reflejadas por sus personajes más destacados, que se corresponden con los bloques temáticos abordados por las diversas categorías de clasificación del ICAA antes citadas.

Para ello, se ha recurrido a una metodología ética u axiológica, pero sin olvidar que el punto de partida de nuestro análisis se sitúa en el lenguaje cinematográfico y en su carácter simbólico. Se suelen enumerar tres estratos en la interpretación de estas metáforas visuales: el material, en el nivel de la trama; el temático y el simbólico (su correspondencia con la historia del arte, de la cultura o de la religión). Nuestro estudio abarca los tres, pues las imágenes expresan ideas, valores o sentimientos que pueden influir en el desarrollo físico, emocional y moral de los jóvenes. Por tanto, todo se ha considerado relevante durante el visionado de los filmes: imagen y texto, lo mostrado y lo implícito, lo denotativo y lo connotativo. Simultáneamente, no hemos perdido de vista el marco axiológico de nuestras conclusiones: los criterios expuestos por el ICAA (que se citan textualmente usando letra negrita en las conclusiones) y no otros referentes o criterios subjetivos.

Obviamente, estas directrices constituyen los presupuestos de nuestra investigación, por lo que no siempre se mostrarán abiertamente en las conclusiones debido a la naturaleza específica de este trabajo: ser un mero recuento y descripción de los incumplimientos de la normativa deontológica sobre calificación de películas y aportar alguna posible solución que evite en un futuro este desajuste ${ }^{3}$.

\subsection{Conclusiones del estudio}

1. Una vez realizado el estudio en función de los principios antes mencionados, se han descubierto 31 películas que no están debidamente calificadas según la normativa del ICAA. En el anexo se especi-

Otra pretensión de este breve estudio es unirse a otros de mayor amplitud, pero de años anteriores y no específicos sobre nuestro país, realizados en el ámbito de la Unión Europea, como el citado en la siguiente nota. 
fican los títulos de esos filmes, junto con la calificación por edades recibida en otros países. A continuación se aportan las razones por las que la calificación no se ajusta a los criterios del ICAA, y la calificación que se propone como más adecuada en función de esos mismos criterios.

2. De las 31 películas detectadas, 12 son de producción española y 19 extranjeras. Un dato significativo si se tiene en cuenta que el número de estrenos anuales de cintas foráneas triplica al de producciones nacionales. En el año 2005, de 6 películas detectadas con una calificación incorrecta, 1 era española. Pero en 2006, de 19 películas detectadas, 9 españolas incumplían la normativa. Y en 2007, de 6 casos analizados, eran 2 las producciones españolas que no se ajustaban a la normativa del ICAA. En definitiva, casi el 50 \% de las películas detectadas eran españolas. Y eso a pesar de que el total de películas que no se ajustan al código sea inferior al 5\% del total de cintas estrenadas en España en esos tres años.

3. Otro dato interesante muestra que la calificación, en 15 casos de los 31 estudiados, difiere esencialmente de la ofrecida por otros países, incluso por algunos que suelen aplicar la normativa de modo poco restrictivo en algunas categorías (como mayores de 16 ó 18 años), como es el caso de Alemania ${ }^{4}$.

4. Y el dato definitivo, que nos permite aportar una posible solución al problema, es que 14 casos de los detectados se pueden resolver introduciendo un pequeño añadido en la normativa del ICAA: estableciendo una nueva categoría, ya existente en muchos países, que establezca que una PELÍCULA NO ESTÁ RECOMENDADA PARA MENORES DE 16 AÑOS (NR 16). Hay una gran diferencia entre el desarrollo emocional y ético de un adolescente de 13 años y el de un joven de 18. Esa laguna podría suplirla esta nueva categoría de NR16, que por otra parte ya existe con esa denominación, u otra similar, en muchos países europeos y en Estados Unidos ${ }^{5}$.

5. En cuanto a los contenidos que han sido motivo de incumplimiento o deficiente aplicación de la normativa, hemos destacado los siguientes:

4 Un informe de la Comisión Europea de mayo de 2003 (Olsberg-SPI, 2003: 83) demuestra, sin embargo, que Francia es uno de los países más liberales (junto con Italia) en la aplicación de los criterios de clasificación por edades y sin embargo, Inglaterra e Irlanda los más estrictos. España está en el sexto lugar por la cola, por delante de Bélgica, Luxemburgo y Grecia. El presidente de la Comisión francesa de calificación de películas se justifica diciendo que en otros países como España la calificación no es vinculante pero en Francia está prohibido (bajo penalización para el cine que lo admita) que vea un filme un menor de la edad establecida en la calificación (Delon, 2003: 5).

5 Se pueden consultar las categorías de clasificación de algunos países como Reino Unido, Francia o Estados Unidos en las siguientes páginas web:[ http://www.bbfc.co.uk/; http://www.cnc.fr/; http://www.mpaa.org/] 
a. Comportamientos sociales: 16 de las películas detectadas presentan alguno de estos contenidos: la venta y el consumo de sustancias (tabaco, alcohol, drogas) que puedan ser perjudiciales para la salud, sobre todo a menores de 18 años. Jóvenes protagonistas que eluden sus responsabilidades o muestran un comportamiento incívico que puede provocar la imitación en adolescentes. En otras se presenta de un modo cómico, complaciente y pesimista, actitudes intolerantes y racistas. También se presentan en algunos de los filmes estudiados, situaciones y manifestaciones sobre una cultura o religión determinadas, en un contexto histórico y geográfico que no permite el distanciamiento del menor de trece años debido a su falta de formación cultural.

b. Temática conflictiva: 14 películas presentan alguno de estos contenidos: muestran de modo explícito la muerte de personajes próximos al entorno familiar o afectivo de un menor, cuando la situación no se resuelve sin provocar angustia; presentan de modo explícito, y sin solución positiva, conflictos exacerbados de carácter religioso; plantean dilemas morales generadores de angustia, por la ausencia de solución positiva y sus consecuencias negativas irreversibles; y aparecen escenas e imágenes basadas en el predominio del miedo y el terror, como si fuera la tónica dominante durante una determinada época o sociedad.

c. La violencia se presenta en 14 filmes, como la única forma de solucionar conflictos: asesinatos, suicidios, torturas, etc. También aparecen graves conflictos emocionales, por ejemplo, la venganza, sin un tratamiento constructivo. Y también se muestran con detalle y como irresolubles, o sólo solucionables por medio de la violencia y el terrorismo, conflictos sociales, raciales y políticos.

d. 23 filmes muestran relaciones afectivo-sentimentales, con manifestaciones sexuales explícitas, sin que el elemento romántico o el tratamiento humorístico o paródico genere un efecto de distanciamiento y atenuación del carácter erótico. En algunos casos esa relación se establece entre un adulto y un menor, o con una gran diferencia de edad entre la pareja (uno de ellos podría ser el hijo del otro). En otros casos se presentan las relaciones sexuales de forma obscena y violenta, con elementos que suponen una degradación de la dignidad humana. La violencia unida al sexo (en concreto la violación) también se muestra en varios casos.

6. Nuestro estudio ha revelado la existencia, dentro del grupo de las 31 películas detectadas, de 8 casos llamativos de incumplimiento de la normativa del ICAA, que procedemos a mencionar y describir:

a. Tapas: presenta la venta y el consumo de sustancias (tabaco, alcohol, drogas) que pueden ser perjudiciales para la salud. Aparece la muerte de un anciano provocada (una especie de eutanasia) por 
su propia mujer. Muestra relaciones afectivo-sentimentales con manifestaciones sexuales explícitas, que en un caso adquieren un tratamiento humorístico o paródico, pero en otros no generan un efecto de distanciamiento y atenuación del carácter erótico, además de mostrar una gran diferencia de edad entre la pareja (uno de ellos podría ser el hijo de la mujer). A pesar de esto la calificación recibida es de NR 7, en lugar de NR 13.

b. Teresa, el Cuerpo de Cristo presenta situaciones y manifestaciones sobre la religión católica en un contexto histórico y geográfico que no permite el distanciamiento del menor de trece años, pues la mirada crítica es algo impensable en un sujeto con la formación cultural propia de la infancia o la pre-adolescencia. Además, la descalificación en el enfoque de ese periodo histórico es total, pues no se trata de un mero informativo sobre un hecho negativo asociado a una cultura o religión determinadas. También refleja actos de tortura no aptos para menores de 13 años, y presenta de modo explícito, y sin solución positiva, conflictos exacerbados de carácter religioso. Plantea dilemas morales generadores de angustia, por la ausencia de solución positiva y sus consecuencias negativas irreversibles, como es el caso de la realización de un aborto a una novicia, mostrado con crudeza, y la aparición de escenas e imágenes basadas en el predominio del miedo y el terror, como si fuera la tónica dominante del Siglo de Oro español. A pesar de esto la calificación recibida es de NR 7, en lugar de NR 13.

c. Alatriste ofrece un tratamiento de la violencia y del sexo que no la hace recomendable para menores de 13 años. Sin embargo, la calificación recibida es de NR 7.

d. El filme Los Borgia presenta graves conflictos emocionales (por ejemplo la venganza, el odio en el seno de la familia y el incesto sugerido) sin un tratamiento constructivo. Se muestra la violencia como la única forma de solucionar conflictos. Y la presencia de relaciones sexuales de forma insistente y obscena, con ausencia de romanticismo o comicidad que provoque distanciamiento. No obstante, la calificación recibida es de NR 13, en lugar de NR 18.

e. María Antonieta muestra algunos comportamientos sociales conflictivos y alguna situación que presenta relaciones afectivas con connotaciones sexuales que no la hacen recomendable para menores de 13 años. Por tanto, no se incluye en los materiales de carácter educativo o pedagógico para la formación sexual. A pesar de esto la calificación recibida es de T, en lugar de NR 7 ó 13.

f. Venus muestra algunos comportamientos sociales conflictivos y alguna situación que presenta relaciones afectivas con connotaciones sexuales entre un adulto y una menor, que no la hacen re- 
comendable para menores de 13 años. Por tanto, no se incluye en los materiales de carácter educativo o pedagógico para la formación sexual. A pesar de esto la calificación recibida es de NR 7, en lugar de NR 13.

g. La vida de los otros muestra algunos comportamientos sociales conflictivos y violentos (incluido el suicidio) y situaciones que presentan relaciones afectivas con connotaciones sexuales (en algunos casos forzadas) que no la hacen recomendable para menores de 13 años. A pesar de esto la calificación recibida es de NR 7, en lugar de NR 13.

h. La carta esférica presenta comportamientos y actitudes, como el arribismo a cualquier precio, inadecuados para el menor de trece años. También presenta situaciones de corrupción institucional (pública o privada) sin un juicio claro sobre su inmoralidad. También muestra la violencia como forma de solucionar los problemas, llevada a cabo por protagonistas o personajes "positivos" que aparecen como autores de actos violentos individuales. Las relaciones de los protagonistas adquieren manifestaciones sexuales explícitas en las que el carácter romántico brilla por su ausencia, y tampoco alcanzan un tratamiento humorístico, o paródico, que genere un efecto de distanciamiento y atenuación del carácter erótico. Y sin embargo, la calificación recibida es de NR 7, en lugar de NR 13.

7. En estos 8 casos da la impresión de que no se ha buscado por encima de todo el bien del espectador, sino el beneficio de las distribuidoras, que han pretendido ampliar el abanico de su mercado de ventas, sobre todo si tenemos en cuenta que al tratarse de películas históricas o adaptaciones literarias, pueden encontrar en el ámbito escolar un fructífero campo para la comercialización.

8. Para evitar situaciones como la precedente, se propone efectuar un control y seguimiento de las normas de calificación de películas del ICAA. Para ello podría ser útil que se firmara un acuerdo similar al realizado para el control de los contenidos televisivos dirigidos a la infancia y la juventud. Las partes adheridas al convenio propuesto de regulación de películas realizarían un seguimiento estrecho de su aplicación, corrigiendo de forma inmediata los posibles incumplimientos que se detecten.

9. Para el seguimiento y control de este código se podría establecer un Comité de Autorregulación compuesto por especialistas en ética y psicología infantil y juvenil, organizaciones representativas de la sociedad civil relacionadas con el objeto de este código (como asociaciones de telespectadores y asociaciones de padres), productores, distribuidores, exhibidores y representantes de la Administración. Su principal función consistiría en emitir dictámenes relativos a las reclamaciones presentadas sobre 
las calificaciones emitidas por la Comisión de Calificación de Películas del ICAA, o de la Comunidad Autónoma competente. Los dictámenes deberán resolver las dudas expuestas en las reclamaciones sobre la adecuación de determinados contenidos a las normas establecidas.

10. El Comité de Autorregulación elaborará un procedimiento para tramitar las dudas que pudieran presentarse y emitir dictámenes relativos a las quejas o reclamaciones presentadas por asociaciones de padres, educadores y espectadores, con implantación en todo el territorio español. El Comité de Autorregulación elaborará este procedimiento para la tramitación de las reclamaciones, sobre todo de cara a la edición en DVD de dichas películas. Esos dictámenes deberán concluir con la recomendación de que se aprecian inconvenientes que deben ser subsanados rectificando la calificación por edades de la película en cuestión. $\mathrm{O}$, por el contrario, concluir que no se aprecian irregularidades y la calificación debe ser mantenida. También se elaborará un informe anual, que será público, sobre los resultados de la aplicación de la normativa. Y debido a la dificultad que presenta el control parental sobre lo exhibido en las salas de cine, se propone una difusión del código a través del medio cinematográfico y televisivo (por ejemplo, en la publicidad previa a la emisión de las películas).

\section{ANEXO. Películas que incumplen la normativa}

Año 2005

\section{TAPAS}

Dirigida por: Juan Cruz, José Corbacho. Calificación: NO RECOM. MENORES DE 7 AÑOS. Nacionalidad: España. Género: Tragicomedia. Intérpretes: Ángel De Andrés, Darío Sánchez, Elvira Mínguez, María Galiana.

CALIFICACIÓN EN OTROS PAÍSES: Noruega: 11 / Argentina: 16 / Holanda: 12 / Brasil: 14.

CALIFICACIÓN PROPUESTAY MOTIVOS DEL INCUMPLIMIENTO: NO RECOMENDADA PARA MENORES DE 13 AÑOS. Los motivos se explican en el artículo.

\section{1:14 DESTINO FATAL}

Dirigida por: Greg Marcks. Calificación: NO RECOM. MENORES DE 13 AÑOS. Nacionalidad: Estados Unidos. Género: Thriller. Intérpretes: Barbara Hershey, Colin Hanks, Hilary Swank, Patrick Swayze.

CALIFICACIÓN EN OTROS PAÍSES: USA: R / Noruega: 15 / Singapur: M18 / Islandia: 16 / Suecia: 15 / Australia: MA / Alemania: 16 / Francia: -12/ Italia: T/ Corea del sur: 15. 


\section{CALIFICACIÓN PROPUESTAY MOTIVOS DEL INCUMPLIMIENTO: NO RECOMENDADA PARA MENORES DE 18 AÑOS.}

Esta calificación se ajusta más a la normativa del ICAA, en concreto en lo referente a los siguientes contenidos:

El tratamiento que 11:14 destino fatal ofrece de los comportamientos sociales, la temática conflictiva y el sexo (elusión de responsabilidades ante un atropello, incitación al crimen, comportamiento incívico que puede provocar la imitación en adolescentes, etc.) requiere una calificación superior a la de no recomendada para menores de 13 años. Pero como en la normativa española no existe la calificación de no recomendada para menores de 16 años, hay que recurrir a la de no recomendada para menores de 18 años, aunque tal vez en este caso sea excesiva, pero la anterior (13 años) se queda corta.

\section{OJALÁ FUERA CIERTO}

Dirigida por: Mark Waters. Calificación: TODOS LOS PUBLICOS. Nacionalidad: Estados Unidos. Género: Comedia Romántica. Intérpretes: Jon Heder, Mark Ruffalo, Reese Whiterspoon.

CALIFICACIÓN EN OTROS PAÍSES: Corea del Sur: 15 / UK: PG / USA: PG-13 / República Checa: 12 / Taiwán: PG-12 / Argentina: Atp / Australia: PG / Holanda: 6 / Filipinas: PG-13 / Suiza: 12 / México: A / Irlanda: PG / Canadá: PG / Hungría: 14.

\section{CALIFICACIÓN PROPUESTAY MOTIVOS DEL INCUMPLIMIENTO: NO RECOMENDADA PARA MENORES} DE 7 AÑOS.

Esta calificación se ajusta más a la normativa del ICAA, en concreto en lo referente a los siguientes contenidos:

Sexo: Ojalá fuera cierto presenta alguna situación y diálogo sexual que presenta relaciones afectivas con connotaciones sexuales. Por tanto no se incluye en los materiales de carácter educativo o pedagógico para la formación sexual.

\section{MANDERLAY}

Dirigida por: Lars Von Trier. Calificación: NO RECOM. MENORES DE 13 AÑOS. Nacionalidad: Dinamarca. Género: Drama. Intérpretes: Bryce Dallas Howard, Danny Glover, Lauren Bacall, Willem Dafoe.

CALIFICACIÓN EN OTROS PAÍSES: Suiza: 14 / Japón: R-18 / Filipinas: R-18 / Brasil: 16 / Italia: VM14 / Finlandia: K-15/ Corea del Sur: 18 / UK: 15 / Singapur: R21 / Portugal: M/16 / Canadá: 14A / Hungría: 16. 


\section{CALIFICACIÓN PROPUESTAY MOTIVOS DEL INCUMPLIMIENTO: NO RECOMENDADA PARA MENORES DE 18 AÑOS.}

Esta calificación se ajusta más a la normativa del ICAA, en concreto en lo referente a los siguientes contenidos:

1. Comportamientos sociales: En Manderlay se presenta de un modo cómico, complaciente y pesimista, actitudes intolerantes y racistas.

2. Temática conflictiva: Se presentan de modo explícito graves conflictos emocionales, por ejemplo la venganza, sin un tratamiento constructivo. Y también se muestran con detalle y como irresolubles, o solo solucionables por medio de la violencia, conflictos sociales y raciales.

3. Sexo: Manderlay presenta las relaciones sexuales de forma obscena y violenta, con elementos que suponen una degradación de la dignidad humana.

\section{DE VENDETTA}

Dirigida por: James Mcteigue. Calificación: NO RECOM. MENORES DE 13 AÑOS. Nacionalidad: Reino Unido. Género: Acción. Intérpretes: Hugo Weaving, John Hurt, Natalie Portman, Stephen Rea.

CALIFICACIÓN EN OTROS PAÍSES: Francia: U (with warning)/ Corea del Sur: 15 / Malasia: 18SG / Australia: MA / Japan: PG-12 / Suiza: 14 / Alemania: 16 / Argentina: 16 / Holanda: 16 / UK: 15 / Finlandia: K-15 / Grecia: K-17 / Portugal: M/16 / Israel: PG / Italia: T / Singapur: M18 / Brasil: 16 / USA: R

CALIFICACIÓN PROPUESTAY MOTIVOS DEL INCUMPLIMIENTO: NO RECOMENDADA PARA MENORES DE 18 AÑOS.

Esta calificación se ajusta más a la normativa del ICAA, en concreto en lo referente a los siguientes contenidos:

Temática conflictiva: $V$ de Vendetta presenta de modo explícito graves conflictos emocionales, como por ejemplo los problemas de identidad sexual, sin un tratamiento constructivo. Y también se muestran con detalle y como irresolubles, o solo solucionables por medio de la violencia y el terrorismo, conflictos sociales y políticos.

\section{MANUALE D'AMORE}

Dirigida por: Giovanni Veronesi. Calificación: NO RECOM. MENORES DE 7 AÑOS. Nacionalidad: Italia. Género: Comedia. Intérpretes: Carlo Verdone, Luciana Littizzetto, Silvio Muccino. 
CALIFICACIÓN EN OTROS PAÍSES: Argentina: 13 / Portugal: 12 / Brasil:14 / Suiza:10.

CALIFICACIÓN PROPUESTAY MOTIVOS DEL INCUMPLIMIENTO: NO RECOMENDADA PARA MENORES DE 13 AÑOS.

Esta calificación se ajusta más a la normativa del ICAA, en concreto en lo referente a los siguientes contenidos:

Temática conflictiva: Manuale d'amore presenta de modo explícito, y sin solución positiva, graves conflictos emocionales en el seno de la familia: el divorcio traumático, etc.

\section{Año 2006}

\section{AZUL OSCURO CASI NEGRO}

Dirigida por: Daniel Sánchez Arévalo. Calificación: NO RECOM. MENORES DE 13 AÑOS. Nacionalidad: España. Género: Comedia dramática. Intérpretes: Quim Gutiérrez, Marta Etura, Antonio de la Torre, Héctor Colomé, Raúl Arévalo, Eva Pallarés.

CALIFICACIÓN EN OTROS PAÍSES: Noruega: 15 / Suiza: 14/ Alemania: 12 / Finlandia: 15.

\section{CALIFICACIÓN PROPUESTAY MOTIVOS DEL INCUMPLIMIENTO: NO RECOMENDADA PARA MENORES} DE 18 AÑOS.

Esta calificación se ajusta más a la normativa del ICAA, en concreto en lo referente a los siguientes contenidos:

1. Temática conflictiva: Azul oscuro casi negro presenta de modo explícito graves conflictos emocionales en el seno de la familia, como los problemas de identidad sexual del padre de uno de los personajes, sin un tratamiento constructivo.

2. Como ocurre con otros casos analizados en este estudio, el tratamiento que Azul oscuro casi negro hace de los comportamientos sociales, la temática conflictiva y el sexo requiere una calificación superior a la de no recomendada para menores de 13 años. Pero como en la normativa española no existe la calificación de no recomendada para menores de 16 años, hay que recurrir a la de no recomendada para menores de 18 años, aunque tal vez en este caso sea excesiva, pero la anterior (13 años) se queda corta. 


\section{TERESA, EL CUERPO DE CRISTO}

Dirigida y escrita por: Ray Loriga. Calificación: NO RECOM. MENORES DE 7 AÑOS. Nacionalidad: España. Género: Drama. Productor: Andrés Vicente Gómez. Intérpretes: Paz Vega, Leonor Watling, Geraldine Chaplin, José Luis Gómez, Eusebio Poncela, Álvaro de Luna, Ángel de Andrés, Manuel Morón.

CALIFICACIÓN EN OTROS PAÍSES: Portugal: M/12

CALIFICACIÓN PROPUESTA Y MOTIVOS DEL INCUMPLIMIENTO: NO RECOMENDADA PARA MENORES DE 13 AÑOS. Los motivos se explican en el artículo.

\section{LA EDUCACIÓN DE LAS HADAS}

Dirigida por: José Luis Cuerda. Calificación: NO RECOM. MENORES DE 7 AÑOS. Nacionalidad: España. Género: Drama. Intérpretes: Ricardo Darín, Irène Jacob, Bebe.

CALIFICACIÓN EN OTROS PAÍSES: Argentina: 13, Portugal: M/12.

CALIFICACIÓN PROPUESTAY MOTIVOS DEL INCUMPLIMIENTO: NO RECOMENDADA PARA MENORES DE 13 AÑOS.

Esta calificación se ajusta más a la normativa del ICAA, en concreto en lo referente a los siguientes contenidos:

El tratamiento que La educación de las hadas hace de los conflictos emocionales en el seno de la familia y del sexo no la hace recomendable para menores de 13 años.

\section{AS IT IS IN HEAVEN (TIERRA DE ÁNGELES)}

Dirigida por: Kay Pollak. Calificación: NO RECOM. MENORES DE 7 AÑOS. Nacionalidad: Suecia. Género: Drama. Intérpretes: Frida Hallgren, Helen Sjoholm, Michael Nyqvist.

CALIFICACIÓN EN OTROS PAÍSES: Suiza: 14; Hong Kong y Hungría: 14; Argentina: 13; Alemania: 12 ; Singapur: no recomendada a menores de 16; Holanda: 12.

CALIFICACIÓN PROPUESTA Y MOTIVOS DEL INCUMPLIMIENTO: NO RECOMENDADA PARA MENORES DE 13 AÑOS.

Esta calificación se ajusta más a la normativa del ICAA, en concreto en lo referente a los siguientes contenidos: 
1. Comportamientos sociales: Tierra de ángeles presenta lenguaje soez, o blasfemo; inadecuado para el menor de trece años.

2. Sexo: Tierra de ángeles insinúa relaciones sexuales que no poseen carácter educativo o pedagógico para la formación sexual, ni están específicamente dirigidas a menores de 13 años.

\section{ALATRISTE}

Dirigida por: Agustín Díaz Yanes. Calificación: NO RECOM. MENORES DE 7 AÑOS. Nacionalidad: España. Género: Aventuras. Intérpretes: Viggo Mortensen, Ariadna Gil, Eduardo Noriega, Unax Ugalde, Antonio Resines.

CALIFICACIÓN EN OTROS PAÍSES: Alemania: 16 / Holanda: 16 / Portugal: M/12 Argentina: 13 / Finlandia: 15 CALIFICACIÓN PROPUESTA Y MOTIVOS DEL INCUMPLIMIENTO: NO RECOMENDADA PARA MENORES DE 13 AÑOS. Los motivos se explican en el artículo.

\section{LA NOCHE DE LOS GIRASOLES}

Dirigida por: Jorge Sánchez-Cabezudo. Calificación: NO RECOM. MENORES DE 13 AÑOS. Nacionalidad: España. Género: Negro. Intérpretes: Carmelo Gómez, Judith Diakhate, Celso Bugallo, Manuel Morón, Vicente Romero.

CALIFICACIÓN EN OTROS PAÍSES: Ireland: 16 / Finland: K-15 / UK: 15

CALIFICACIÓN PROPUESTAY MOTIVOS DEL INCUMPLIMIENTO: NO RECOMENDADA PARA MENORES DE 18 AÑOS.

Esta calificación se ajusta más a la normativa del ICAA, en concreto en lo referente a los siguientes contenidos:

Como ocurre con otros casos analizados en este estudio, el tratamiento que La noche de los girasoles hace de los comportamientos sociales y de la violencia unida al sexo (en concreto una violación) requiere una calificación superior a la de no recomendada para menores de 13 años. Pero como en la normativa española no existe la calificación de no recomendada para menores de 16 años, hay que recurrir a la de no recomendada para menores de 18 años, aunque tal vez en este caso sea excesiva, pero la anterior (13 años) se queda corta. 


\section{SALVADOR PUIG ANTICH}

Dirigida por: Manuel Huerga. Calificación: NO RECOM. MENORES DE 13 AÑOS.

Nacionalidad: España. Género: Drama. Intérpretes: Daniel Brühl, Tristán Ulloa, Leonor Watling, Joel Joan, Ingrid Rubio, Leonardo Sbaraglia.

CALIFICACIÓN EN OTROS PAÍSES: Alemania: 16

\section{CALIFICACIÓN PROPUESTAY MOTIVOS DEL INCUMPLIMIENTO: NO RECOMENDADA PARA MENORES DE 18 AÑOS.}

Esta calificación se ajusta más a la normativa del ICAA, en concreto en lo referente a los siguientes contenidos:

Como ocurre con otros casos analizados en este estudio, el tratamiento que Salvador Puig Antich hace de los comportamientos sociales, la violencia y el sexo requiere una calificación superior a la de no recomendada para menores de 13 años. Pero como en la normativa española no existe la calificación de no recomendada para menores de 16 años, hay que recurrir a la de no recomendada para menores de 18 años, aunque tal vez en este caso sea excesiva, pero la anterior (13 años) se queda corta.

\section{LOS BORGIA}

Dirigida por: Antonio Hernández. Calificación: NO RECOM. MENORES DE 13 AÑOS. Nacionalidad: España. Género: Biográfica. Intérpretes: Eloy Azorín, Luis Homar, María Valverde, Sergio Peris Mencheta.

CALIFICACIÓN EN OTROS PAÍSES: Portugal: Mayores de 16.

CALIFICACIÓN PROPUESTA Y MOTIVOS DEL INCUMPLIMIENTO: NO RECOMENDADA PARA MENORES DE 18 AÑOS. Los motivos se explican en el artículo.

\section{LO QUE SÉ DE LOLA}

Dirigida por: Javier Rebollo. Calificación: NO RECOM. MENORES DE 13 AÑOS.

Nacionalidad: España. Género: Drama. Intérpretes: Lola Dueñas, Michaël Abiteboul, Carmen Machi, Lucienne Deschamps.

CALIFICACIÓN PROPUESTA Y MOTIVOS DEL INCUMPLIMIENTO: NO RECOMENDADA PARA MENORES DE 18 AÑOS. 
Esta calificación se ajusta más a la normativa del ICAA, en concreto en lo referente a los siguientes contenidos:

Como ocurre con otros casos analizados en este estudio, el tratamiento que Lo que sé de Lola hace de la temática conflictiva y el sexo requiere una calificación superior a la de no recomendada para menores de 13 años. Pero como en la normativa española no existe la calificación de no recomendada para menores de 16 años, hay que recurrir a la de no recomendada para menores de 18 años, aunque tal vez en este caso sea excesiva, pero la anterior (13 años) se queda corta.

\section{LOS FANTASMAS DE GOYA}

Dirigida por: Milos Forman. Calificación: NO RECOM. MENORES DE 13 AÑOS. Nacionalidad: España. Género: Drama. Intérpretes: Javier Bardem, Natalie Portman, Stellan Skarsgard.

CALIFICACIÓN EN OTROS PAÍSES: Finlandia: K-13 / Argentina: 13 / Italy: T/ Brasil: 14 / Irlanda: 15A / Suecia: 15 / USA: R / Corea del Sur: 15

\section{CALIFICACIÓN PROPUESTAY MOTIVOS DEL INCUMPLIMIENTO: NO RECOMENDADA PARA MENORES DE 18 AÑOS.}

Esta calificación se ajusta más a la normativa del ICAA, en concreto en lo referente a los siguientes contenidos:

Como ocurre con otros casos analizados en este estudio, el tratamiento que Los Fantasmas de Goya hace de la temática conflictiva (por ejemplo la venganza, el odio) sin un tratamiento constructivo, el sexo y la violencia (se presenta ésta como la única forma de solucionar conflictos), requiere una calificación superior a la de no recomendada para menores de 13 años. Pero como en la normativa española no existe la calificación de no recomendada para menores de 16 años, hay que recurrir a la de no recomendada para menores de 18 años, aunque tal vez en este caso sea excesiva, pero la anterior (13 años) se queda corta.

\section{BABEL}

Dirigida por: Alejandro González Iñarritu. Calificación: NO RECOM. MENORES DE 13 AÑOS. Nacionalidad: Estados Unidos. Género: Thriller. Intérpretes: Brad Pitt, Cate Blanchett, Gael García Bernal

CALIFICACIÓN EN OTROS PAÍSES: Nueva Zelanda: R16 / Portugal: M/12 / France: U / Argentina: 18 / UK: 15 / USA: R / Dinamarca: 15 / Noruega: 15 / Australia: MA / Alemania: 16 / Holanda: 16 / Canadá: 18A / Singapur: M18 / Irlanda: 16 / Japón: PG-12/ Brasil: 16 / Malasia: 18PL / Corea del Sur: 18 / Sudáfrica: 16 / Irán: 18+ / Finlandia: K-15 


\section{CALIFICACIÓN PROPUESTAY MOTIVOS DEL INCUMPLIMIENTO: NO RECOMENDADA PARA MENORES DE 18 AÑOS.}

Esta calificación se ajusta más a la normativa del ICAA, en concreto en lo referente a los siguientes contenidos:

Como ocurre con otros casos analizados en este estudio, el tratamiento que Babel hace de los comportamientos sociales (consumo de drogas y alcohol, sin una visión crítica para el menor), la violencia (se presenta ésta como la única forma de solucionar conflictos), y el sexo (ejercido por menores), requiere una calificación superior a la de no recomendada para menores de 13 años. Pero como en la normativa española no existe la calificación de no recomendada para menores de 16 años, hay que recurrir a la de no recomendada para menores de 18 años, aunque tal vez en este caso sea excesiva, pero la anterior (13 años) se queda corta.

\section{MARIA ANTONIETA}

Dirigida por: Sofia Coppola. Calificación: TODOS LOS PÚBLICOS. Nacionalidad: Estados Unidos. Género: Drama Histórico. Intérpretes: Kirsten Dunst, Asia Argento, Jason Schawartzman, Rip Torn.

CALIFICACIÓN EN OTROS PAÍSES: Argentina: Atp / Taiwan: PG-12 / Australia: PG / Corea del Sur: 15 / Singapur: PG / Canadá: PG / Brasil: 14 / Holanda: AL / USA: PG-13 / Perú: PT / Suiza: 10 / Portugal: M/12 / UK: 12A / Alemania: o.Al. / Finlandia: S / Francia: U / Irlanda: 12A / Filipinas: R-13

CALIFICACIÓN PROPUESTA Y MOTIVOS DEL INCUMPLIMIENTO: NO RECOMENDADA PARA MENORES DE 7 o de 13 AÑOS. Los motivos se explican en el artículo.

\section{EL ÚLTIMO REY DE ESCOCIA}

Dirigida por: Kevin Macdonald. Calificación: NO RECOM. MENORES DE 13 AÑOS. Nacionalidad: Estados Unidos. Género: Drama Histórico. Intérpretes: Forest Whitaker, Gillian Anderson, James Mcavoy.

CALIFICACIÓN EN OTROS PAÍSES: Malasia: 18PL / Argentina: 16 / UK: 15 / Singapur: M18 / USA: R / Canadá: 18A / New Zelanda: R16 / Suiza: 16 / Sudáfrica: 16 / Holanda: 16 / Corea del Sur: 18 / Francia: -12 / Noruega: 15 / Japón: R-15 / Irlanda: 15A / Portugal: M/16 / Finlandia: K-15 / Brasil: 16 / Alemania: 16 CALIFICACIÓN PROPUESTAY MOTIVOS DEL INCUMPLIMIENTO: NO RECOMENDADA PARA MENORES DE 18 AÑOS. 
Esta calificación se ajusta más a la normativa del ICAA, en concreto en lo referente a los siguientes contenidos:

Como ocurre con otros casos analizados en este estudio, el tratamiento que El último rey de Escocia hace de los comportamientos sociales (consumo de drogas y alcohol, sin una visión crítica para el menor), la violencia (se presenta ésta como la única forma de solucionar conflictos: asesinatos, torturas, etc.), y el sexo, requiere una calificación superior a la de no recomendada para menores de 13 años. Pero como en la normativa española no existe la calificación de no recomendada para menores de 16 años, hay que recurrir a la de no recomendada para menores de 18 años, aunque tal vez en este caso sea excesiva, pero la anterior (13 años) se queda corta.

\section{DIARIO DE UN ESCÁNDALO}

Dirigida por: Richard Eyre. Calificación: NO RECOM. MENORES DE 13 AÑOS. Nacionalidad: Reino Unido. Género: Drama. Intérpretes: Cate Blanchett, Judi Dench.

CALIFICACIÓN EN OTROS PAÍSES: Corea del Sur: 18 / Canadá: 14A / USA: R / Suiza: 14 / Australia: MA / Alemania: 12 / Irlanda: 16 / UK: 15 / Argentina: 16 / Nueva Zelanda: M / Singapur: M18 | Portugal: M/16 / Malaysia: 18PL / Holanda: 12 / Finlandia: K-15 / Japón: R-15 / Brasil: 14

CALIFICACIÓN PROPUESTAY MOTIVOS DEL INCUMPLIMIENTO: NO RECOMENDADA PARA MENORES DE 18 AÑOS.

Esta calificación se ajusta más a la normativa del ICAA, en concreto en lo referente a los siguientes contenidos:

Como ocurre con otros casos analizados en este estudio, el tratamiento que Diario de un escándalo hace de los comportamientos sociales y conflictivos relacionados con el sexo (ejercido por menores) requiere una calificación superior a la de no recomendada para menores de 13 años. Pero como en la normativa española no existe la calificación de no recomendada para menores de 16 años, hay que recurrir a la de no recomendada para menores de 18 años, aunque tal vez en este caso sea excesiva, pero la anterior (13 años) se queda corta.

\section{VENUS}

Dirigida por: Roger Michell. Calificación: NO RECOM. MENORES DE 7 AÑOS. Nacionalidad: Reino Unido. Género: Comedia. Intérpretes: Leslie Phillips, Peter O'Toole, Richard Griffiths, Vanessa Redgrave. 
CALIFICACIÓN EN OTROS PAÍSES: Finlandia: K-11 / UK: 15 / Australia: MA / Singapur: NC-16 / Suecia: 7 / USA: R / Argentina: 13 / Portugal: M/12 / Irlanda: 16 / Holanda: 6

CALIFICACIÓN PROPUESTAY MOTIVOS DEL INCUMPLIMIENTO: NO RECOMENDADA PARA MENORES DE 13 AÑOS. Los motivos se explican en el artículo.

\section{RETRATO DE UNA OBSESIÓN}

Dirigida por: Steven Shainberg. Calificación: NO RECOM. MENORES DE 13 AÑOS. Nacionalidad: Estados Unidos. Género: Drama. Intérpretes: Nicole Kidman, Harris Yulin, Jane Alexander, Robert Downey Jr.

CALIFICACIÓN EN OTROS PAÍSES: Hong Kong: IIB / Argentina: 16 / Grecia: K-13 / Finlandia: K-13 / Japón: R-18 / USA: R / Portugal: M/16 / Irlanda: 15A / Singapur: M18 / Holanda: 12 / Suecia: 7 / Chile: 14 / UK: 15 / Corea del Sur: 18

\section{CALIFICACIÓN PROPUESTAY MOTIVOS DEL INCUMPLIMIENTO: NO RECOMENDADA PARA MENORES DE 18 AÑOS.}

Esta calificación se ajusta más a la normativa del ICAA, en concreto en lo referente a los siguientes contenidos:

Como ocurre con otros casos analizados en este estudio, el tratamiento que Retrato de una obsesión hace de los comportamientos sociales, conflictivos y sexuales, en algunos casos degradantes para la dignidad humana, requiere una calificación superior a la de no recomendada para menores de 13 años.

\section{¡CÁSATE CONMIGO!}

Dirigida por: Michael Ian Black. Calificación: TODOS LOS PÚBLICOS. Nacionalidad: Estados Unidos. Género: Comedia. Intérpretes: Jason Biggs, Isla Fisher, Joanna Gleason, Joe Pantoliano.

CALIFICACIÓN EN OTROS PAÍSES: Corea del Sur: 15 / Taiwan: R-12 / Alemania: 12 / Irlanda: 15A / USA: R / Holanda: 6 / Singapur: NC-16 / UK: 15

\section{CALIFICACIÓN PROPUESTAY MOTIVOS DEL INCUMPLIMIENTO: NO RECOMENDADA PARA MENORES} DE 7 o de 13 AÑOS.

Esta calificación se ajusta más a la normativa del ICAA, en concreto en lo referente a los siguientes contenidos: ¡Cásate conmigo! muestra algunos comportamientos sociales conflictivos y algunas situaciones que presentan relaciones afectivas con connotaciones sexuales que no la hacen recomendable para menores de 13 
años. Por tanto, no se incluye en los materiales de carácter educativo o pedagógico para la formación sexual.

\section{IRINA PALM}

Dirigida por: Sam Garbarski. Calificación: NO RECOM. MENORES DE 13 AÑOS. Nacionalidad: Alemania, Bélgica, Francia, Luxemburgo, Reino Unido. Género: Drama. Intérpretes: Marianne Faithfull, Miki Manojlovic.

CALIFICACIÓN EN OTROS PAÍSES: Alemania: 12 / Portugal: M/16 / Suiza: 14 / Holanda: 12 / Italia: T / USA: R / Finlandia: K-15 / Corea del Sur: 18 / Singapur: M18 / Japón: R-15

\section{CALIFICACIÓN PROPUESTAY MOTIVOS DEL INCUMPLIMIENTO: NO RECOMENDADA PARA MENORES DE 18 AÑOS.}

Esta calificación se ajusta más a la normativa del ICAA, en concreto en lo referente a los siguientes contenidos:

Como ocurre con otros casos analizados en este estudio, el tratamiento que Irina Palm hace de los comportamientos sociales y conflictivos relacionados con el sexo requiere una calificación superior a la de no recomendada para menores de 13 años. Pero como en la normativa española no existe la calificación de no recomendada para menores de 16 años, hay que recurrir a la de no recomendada para menores de 18 años, aunque tal vez en este caso sea excesiva, pero la anterior (13 años) se queda corta.

\section{THIS IS ENGLAND}

Dirigida por: Shane Meadows. Calificación: NO RECOM. MENORES DE 13 AÑOS. Nacionalidad: Reino Unido. Género: Drama. Intérpretes: Thomas Turgoose, Stephen Graham.

CALIFICACIÓN EN OTROS PAÍSES: Alemania: 12 / Australia: MA / Finlandia: K-15 / UK: 18 / France: U (with warning) / Irlanda: 18 / Suecia: 11

\section{CALIFICACIÓN PROPUESTA Y MOTIVOS DEL INCUMPLIMIENTO: NO RECOMENDADA PARA MENORES DE 18 AÑOS.}

Esta calificación se ajusta más a la normativa del ICAA, en concreto en lo referente a los siguientes contenidos:

Como ocurre con otros casos analizados en este estudio, el tratamiento que This is England hace de los comportamientos sociales conflictivos y violentos (en los que se ve envuelto el menor protagonista) requiere una calificación superior a la de no recomendada para menores de 13 años. Pero como en la 
normativa española no existe la calificación de no recomendada para menores de 16 años, hay que recurrir a la de no recomendada para menores de 18 años, aunque tal vez en este caso sea excesiva, pero la anterior (13 años) se queda corta.

Aก̃ 2007

\section{LA VIDA DE LOS OTROS}

Dirigida por: Florian Heckel Von Donnersmarck. Calificación: NO RECOM. MENORES DE 7 AÑOS. Nacionalidad: Alemania. Género: Drama. Intérpretes: Martina Gedeck, Ulrich Mühe.

CALIFICACIÓN EN OTROS PAÍSES: Australia: MA / Suiza: 10 / Argentina: 13 / Italia: T / Finlandia: K-11 / Malasia: U / Singapur: M18 / Corea del Sur: 15 / Canadá: 14 / Irlanda: 15A / Suecia: 7 / Holanda: 12 / Portugal: M/16 / Alemania: 12 / USA: R / República Checa: 12 / UK: 15

CALIFICACIÓN PROPUESTAY MOTIVOS DEL INCUMPLIMIENTO: NO RECOMENDADA PARA MENORES DE 13 AÑOS. Los motivos se explican en el artículo.

\section{EL JEFE DE TODO ESTO}

Dirigida por: Lars Von Trier. Calificación: NO RECOM. MENORES DE 7 AÑOS. Nacionalidad: Dinamarca. Género: Comedia. Intérpretes: Fridrik Thor Fridriksson, Jens Albinus, Iben Hjejle, Peter Gantzler.

CALIFICACIÓN EN OTROS PAÍSES: Argentina: 13 / Brasil: 16 / Italia:VM14 (re-rating) / Australia: MA / Singapur: M18 / Corea del sur: 18 / Suiza: 10 / Holanda: 6

CALIFICACIÓN PROPUESTA Y MOTIVOS DEL INCUMPLIMIENTO: NO RECOMENDADA PARA MENORES DE 13 AÑOS.

Esta calificación se ajusta más a la normativa del ICAA, en concreto en lo referente a los siguientes contenidos:

Sexo: El jefe de todo esto presenta una escena sexual (incluido sexo oral y anal) que aunque tenga cierto tratamiento humorístico, por tratarse de una comedia, es demasiado explícita para un menor de 13 años, pues no llega a generar totalmente un efecto de distanciamiento que atenúe su carácter erótico. Además, la escena se sitúa en un ámbito laboral, lo que sugiere la permisión del acoso sexual en el lugar de trabajo. Una situación que el menor no puede apreciar en su contexto ni, por tanto, ejercer un juicio crítico. 


\section{EL BUEN PASTOR}

Dirigida por: Robert De Niro. Calificación: NO RECOM. MENORES DE 13 AÑOS. Nacionalidad: Estados Unidos. Género: Drama. Intérpretes: Matt Damon, Alec Baldwin, Angelina Jolie, Robert De Niro.

CALIFICACIÓN EN OTROS PAÍSES: Malasia: 18PL / Italia: T / Holanda: 16 / Finlandia: K-15 / Irlanda: 15A / Suiza: 12 / Suecia: 15 / Noruega: 15 / Filipinas: R-13 / UK: 15 / Canadá: 13+ / Corea del Sur: 18 / Alemania: 12 / Nueva Zelanda: M / Singapur: M18 / Australia: M / USA: R / Dinamarca: 15 / Portugal: M/12 / Argentina: 13 / Brasil: 14 / Canadá: 14A

\section{CALIFICACIÓN PROPUESTAY MOTIVOS DEL INCUMPLIMIENTO: NO RECOMENDADA PARA MENORES DE 18 AÑOS.}

Esta calificación se ajusta más a la normativa del ICAA, en concreto en lo referente a los siguientes contenidos:

Como ocurre con otros casos analizados en este estudio, el tratamiento que El buen pastor hace de los comportamientos sociales conflictivos y violentos (torturas, asesinatos y suicidios) requiere una calificación superior a la de no recomendada para menores de 13 años. Pero como en la normativa española no existe la calificación de no recomendada para menores de 16 años, hay que recurrir a la de no recomendada para menores de 18 años, aunque tal vez en este caso sea excesiva, pero la anterior (13 años) se queda corta.

\section{RIVALES CASI IGUALES}

Dirigida por: Miguel Ángel Calvo Buttini. Calificación: TODOS LOS PUBLICOS. Nacionalidad: España. Género: Comedia. Intérpretes: Andoni Gracia, Txema Blasco, Teresa Gimpera, Merce Llorens, María Galiana.

CALIFICACIÓN PROPUESTA Y MOTIVOS DEL INCUMPLIMIENTO: NO RECOMENDADA PARA MENORES DE 7 AÑOS.

Esta calificación se ajusta más a la normativa del ICAA, en concreto en lo referente a los siguientes contenidos:

2 rivales casi iguales muestra algunos comportamientos, actitudes y costumbres ininteligibles para el menor de siete años y que le pueden crear desconcierto. En concreto, alude a conflictos de orden sexual que afectan al entorno familiar de un menor y que requieren un mínimo (re)conocimiento de su existencia por el menor de siete años, por lo que pueden afectar negativamente a la comprensión de su entorno habitual. 


\section{ODETTE. UNA COMEDIA SOBRE LA FELICIDAD}

Dirigida por: Eric-Emmanuel Schmidt. Calificación: TODOS LOS PÚBLICOS. Nacionalidad: Francia. Género: Comedia. Intérpretes: Catherine Frot, Albert Dupontel, Jacques Weber.

CALIFICACIÓN EN OTROS PAÍSES: Suiza: 10 / Alemania: 6 / Holanda: AL

\section{CALIFICACIÓN PROPUESTAY MOTIVOS DEL INCUMPLIMIENTO: NO RECOMENDADA PARA MENORES} DE 7 AÑOS.

Esta calificación se ajusta más a la normativa del ICAA, en concreto en lo referente a los siguientes contenidos:

Odette. Una comedia sobre la felicidad muestra algunos comportamientos, actitudes y costumbres ininteligibles para el menor de siete años y que le pueden crear desconcierto. En concreto, alude a conflictos de orden sexual que afectan al entorno familiar de un menor y que requieren un mínimo (re)conocimiento de su existencia por el menor de siete años, por lo que pueden afectar negativamente a la comprensión de su entorno habitual.

\section{LA CARTA ESFÉRICA}

Dirigida por: Imanol Uribe. Calificación: NO RECOM. MENORES DE 7 AÑOS. Nacionalidad: España. Género: Aventuras. Intérpretes: Darío Grandinetti, Aitana Sánchez-Gijón, Enrico Lo Verso, Carmelo Gómez. Argumento: Arturo Pérez Reverte.

CALIFICACIÓN PROPUESTAY MOTIVOS DEL INCUMPLIMIENTO: NO RECOMENDADA PARA MENORES DE 13 AÑOS. Los motivos se explican en el artículo.

\section{Referencias bibliográficas}

Arza, J. (2008): Familia, televisión y otras pantallas, Consejo Audiovisual de Navarra.

Delon, F. (2003): The French experience: from censorship to classification. Chairman of the French Commission of film classification. Disponible en Internet [URL] http://www.classification.gov.au/resource.html?resource=253\&filename=253.pdf Marías, J. (1992): La educación sentimental, Madrid: Alianza.

Muñoz, J. J. (2005): De Casablanca a Solas. La creatividad ética en cine y televisión, Madrid: Ediciones Internacionales Universitarias. 
Olsberg-SPI y KEA European Affairs in association with KPMG (2003): Empirical Study on the Practice of the Rating of Films Distributed in Cinemas, Television, DVD and Videocassettes in the EU and EEA Member States. Disponible en Internet [URL] http://ec.europa.eu/avpolicy/docs/library/studies/finalised/studpdf/rating_finalrep2.pdf

Pardo, A. (2001): “El cine como medio de comunicación y la responsabilidad social del cineasta”, en Codina, M. (ed.): De la ética desprotegida, Pamplona: Eunsa, pp.117-141.

Pérez Tornero, J.M. (2003): “Libro Blanco: La educación en el entorno audiovisual”, Quaderns del CAC. Número extraordinario, Noviembre 2003.

Tur, V. (2006): Calidad de los contenidos audiovisuales infantiles, Ministerio de Educación y Ciencia-FEDER. Secretaría de Humanidades y Ciencias Sociales. Programa I+D+i 2004-2007. Autores: Macarena Lozano Oyola y Luis Benjamín Romero Landa. Disponible en Internet [URL] http://www.ua.es/es/grupo/comunicacioneinfancia/documentos/doc_grupo_invest /informes/informe-sobre-experiencias-de-certificaci-n.pdf

Página web del ICAA. Disponible en Internet [URL] http://www.mcu.es/bbddpeliculas/buscarPeliculas.do

Página web de British Board of Film Classification (BBFC). Disponible en Internet [URL] http://www.bbfc.co.uk/

Página web de Film Classification Committee del CNC. Disponible en Internet [URL] http://www.cnc.fr/

Página web de Motion Picture Association of America (MPAA). Disponible en Internet [URL] http://www.mpaa.org 